\title{
Absolute Chiral Recognition with Hybrid Wireless Electrochemical Actuators
}

Serena Arnaboldi1,3‡, Bhavana Gupta1‡, Tiziana Benincori2, Giorgia Bonetti2, Roberto Cirilli4, Alexander Kuhn1*

1 Univ. Bordeaux, CNRS UMR 5255, Bordeaux INP, ENSCBP, 16 avenue Pey Berland, 33607 Pessac, France,

*kuhn@enscbp.fr

2 Univ.degli Studi dell'Insubria, Dip. di Scienza e Alta Tecnologia,Via Valleggio 11, 22100 Como, Italy

3 Univ. degli Studi di Milano, Dip. di Chimica, Via Golgi 19, 20133 Milano; Italy

4 Centro Nazionale per il Controllo e la Valutazione dei Farmaci, Istituto Superiore di Sanità, Viale Regina Elena 299, 00161 Roma, Italy

KEYWORDS: chiral recognition $\bullet$ bipolar electrochemistry $\bullet$ actuator $\bullet$ conducting polymers $\bullet$ enantiomeric excess

ABSTRACT: Chiral discrimination is of crucial importance for many applications, including drug cross checking and electronic tongue type devices. In a typical sensing scheme, an enantiomeric selector is combined with an appropriate transduction mecha-nism. We propose here a hybrid material composed of an electrically conducting oligomer i.e. oligo-(3, 3'-dibenzothiophene) bear-ing inherently chiral features, and polypyrrole as a support which can undergo electrochemical actuation. The combination of both leads to a freestanding film that is addressable in a wireless way based on the principle of bipolar electrochemistry. The induced redox reactions lead to wellpronounced actuation when DOPA with the right chirality is present in solution as a model analyte, whereas absolutely no electromechanical response is measured for the wrong enantiomer. This constitutes a straightforward and absolute read out of chiral information where the amplitude of actuation is correlated with the concentration of the analyte. Opti-mization of the scheme results in highly efficient bending, and thus opens up new directions in the field of chiral technologies.

Chiral discrimination is of vital importance in analytical chemistry as it is highly desired, among others, in the pharma-ceutical industry and forensic or environmental science to test analytes of pharmaceutical and biological interest.1-6 In the case of chiral drugs, often only one enantiomer is effective and useful for the consumer, whereas the antipode is inactive or even can have detrimental effects. Therefore, selective recognition of enantiomers is the basis of both, drug produc-tion and quality control.5, 7-8 Among all the different analytical approaches proposed in this context, electrochemical ones look potentially very attractive, with intrinsic advantages including selectivity, sensitivity, low cost and easy transduc-tion of recognition events and signal processing.9-11 In this context it is very interesting to combine electrochemistry with chirality in order to achieve a high degree of selectivity and sensitivity. Recently, certain oligomers in which the molecular function responsible for electroconductivity coincides with the 3D molecular scaffold responsible for chirality (inherently chiral oligomers) emerged as a new tool for chiral voltamme-try. In this case, differentiation of the enantiomers of a chiral analyte is possible in terms of variations of peak potential values, rather than measuring different current intensities enabling effective enantiorecognition.12 When this feature is combined with a linear dynamic range for currents it is also possible to determine enantiomeric excesses in mixed solu-tions, containing different ratios of antipodes of a chiral probe. In fact, during oligomerization, the inherent chirality of the oligomers is translated into helical foldamer-like macro- or supramolecular structures.13 The obtained oligomers are easy to prepare and highly robust in comparison with other materi-als investigated for 
implementation of chirality on electrode surfaces. Their advantages with respect to other approaches used to generate enantioselective surfaces have been de-scribed in detail in the literature.1 Recently Arnaboldi et al. demonstrated that enantiopure inherently chiral oligomers with an atropisomeric core (e.g., 3,3'-bibenzothiophene,12 2,2'-biindole13 and 3,3'-bithiophene14) allow recognizing chiral probes with different chemical nature in terms of difference in formal electrochemical potential values, combined with the possibility, in some cases, to determine enantiomeric excess. 15 The perspective of this concept, and the aim of the present contribution, is to develop low cost, portable and wireless transducers for a quick and absolute detection of chiral infor-mation. Designing a wireless readout scheme of chiral infor-mation requires two essential ingredients: one for the enanti-oselective electron transfer process and the other one for transduction. To simplify the system, a wireless mode of inter-facing has recently been considered to facilitate electrochemi-cal detection, especially when having in mind the increasing popularity of smartphones combined with a portable potenti-ostat.16-17 However, for classic electrochemical transduction, the detector electrode has to be wired to the power supply. In contrast, when using a bipolar electrochemistry mode, an electric field is generated between two feeder electrodes to induce a polarization of a remotely placed bipolar electrode which undergoes the electrochemical transformation. The current passing through the bipolar electrode as a function of the concentration of electroactive molecules can be evaluated with different approaches such as electrochemiluminescence, electrodissolution, concentration enrichment of charged ana-lytes and electromechanical deformation etc.18-31 The optical readout of electrochemical activity provides a low cost meas-urement of different species and has various other advantages like the easy detection of color changes or light emission, eventually with the naked eye, useful for chemical imaging and in the frame of massive parallel analytical arrays.32-36 Apart from light emission, colour changes and fluorescence, mechanical deformation is an interesting alternative for the optical visualization of signals. It has been recently proposed in combination with bipolar electrochemistry as a straightforward concept in analytical chemistry.19 To achieve bipolar electrochemical processes endowed with enantioselectivity, chiral imprinted mesoporous platinum was combined with polypyrrole in a hybrid film, allowing the preferential reaction of one enantiomer, detected by a differential electromechanical deformation of the polypyrrole used as a freestanding actuator.37 The relative difference of bending was used as a sign of enantioselectivity, but does not allow absolute discrim-ination of the two antipodes. In this context, a material, on which the enantiomers of a given chiral probe undergo elec-tron transfer at two well-separated potentials when used as a bipolar electrode, could lead to a tool for absolute chiral recognition. As the above described oligomers reveal a ther-modynamically different interaction with the two enantiomers of an analyte, combining them with polypyrrole as an actuator material should lead to an absolute electromechanical readout of chiral information. Such a yes/no answer, combined with a quantitative concentration measurement would greatly en-hance the possible applications of this wireless approach. We report here the synthesis of a freestanding hybrid polymer film by electrodepositing an inherently chiral enantiopure monomer with a 3,3'bibenzothiophene core (i.e. 2,2'-bis[2-(5,2'-bithienyl)]-3,3'-bithianaphthene, named BT2T4, Scheme 1) on polypyrrole (Ppy), which is electromechanically active, in order to combine chiral electrochemical recognition with an easy optical read out of the simultaneously occurring actua-tion. This proof-of-concept set-up is tested for the absolute discrimination of the two enantiomers of a chiral probe i.e. L- or D-DOPA.

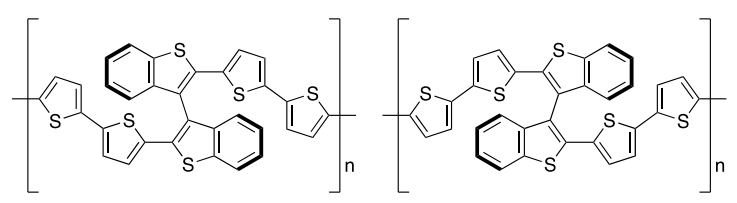


Scheme 1. Chemical structures of the two enantiomers of the BT2T4 oligomers

\section{EXPERIMENTAL SECTION}

Synthesis of the polypyrrole film. For the synthesis of polypyr-role a solution of pyrrole monomer ( 0.2 M) (Sigma Aldrich) was dissolved in Milli Q water with dodecylbenzene sulfonate (DBS) (0.25 M) (Sigma Aldrich). After complete dissolution of both components, two gold coated glass slides were positioned parallel in a beaker at a distance of $1.5 \mathrm{~cm}$. The beaker was filled with $12 \mathrm{~cm} 3$ of this solution and one gold coated glass was used as a working electrode while the other one as counter electrode and $\mathrm{Ag} / \mathrm{AgCl}(3 \mathrm{M} \mathrm{KCl})$ was the refer-ence electrode. A fixed current of $0.004 \mathrm{~A}$ was applied for $1.5 \mathrm{~h}$ for the polymerization of pyrrole. The charge consumed during polymeri-zation is around 20 C. After polymerization, the polymer coated substrate was washed with water, dried and used for further oligomer-ization of chiral monomer i.e. 2,2'-bis[2-(5,2'-bithienyl)]-3,3'-bithianaphthene.

Electrosynthesis of enantiopure oligo-(R)- or oligo-(S)-2,2'-bis[2-(5,2'-bithienyl)]-3,3'-bithianaphthene (oligo-(R)- or (S)-BT2T4). The electrosynthesis of enantiopure oligo-(S)-BT2T4 and oligo-(R)-BT2T4 was carried out with the polypyrrole substrate acting as working electrode in a small beaker containing 5 $\mathrm{cm} 3$ of $0.1 \mathrm{M}$ solution of lithium perchlorate ( $\mathrm{LiClO} 4)$ in acetonitrile (MeCN) and the (R)- or (S)enantiopure monomers at $5 \mathrm{mM}$ concentration. The counter electrode was a platinum grid located at $1.5 \mathrm{~cm}$ from the working electrode together with an $\mathrm{Ag} / \mathrm{AgCl}$ reference electrode. Oligo-(S)-BT2T4 and oligo-(R)-BT2T4 were synthetized by chronopo-tentiometry at a fixed current value of $0.002 \mathrm{~A}$ for 40 minutes. After deposition of the oligo-(3,3'dibenzothiophene)-polypyrrole hybrid films, they were peeled off from the gold electrode and then properly cut to be used as actuators for the bipolar chiral recognition experi-ments. The total length of the bipolar electrode is $1.1 \mathrm{~cm}, 3 \mathrm{~mm}$ of which correspond to the actuator part. In order to maximize the cur-rent density on the actuator section, the oligo-BT2T4 covered end is $5 \mathrm{~mm}$ in width while the naked Ppy is $1 \mathrm{~mm}$.

Differential pulse voltammetry (DPV) experiments. DPV exper-iments were carried out in a beaker, used as electrochemical cell, containing the enantiomers of L- or D-DOPA ( $5 \mathrm{mM}$ ) dissolved in water and $0.2 \mathrm{M} \mathrm{LiClO4}$. The reference electrode was $\mathrm{Ag} / \mathrm{AgCl}$ and a platinum grid the counter electrode. The working electrode was a hybrid electrode composed of an oligo-(S)-BT2T4 layer, deposited on a freestanding Ppy film. This film was carefully isolated on the back-side with varnish and connected with a copper tape to the potentiostat. The optimized DPV parameters used for recording the voltammetric signals of L- or D-DOPA were: step potential $10 \mathrm{mV}$, modulation amplitude $60 \mathrm{mV}$, modulation time $40 \mathrm{~ms}$, and interval time $200 \mathrm{~ms}$.

Bipolar chiral recognition. For bipolar chiral recognition, enanti-opure (R)- or (S)-oligo-(3,3'dibenzothiophene)-polypyrrole hybrid films $(1.1 \mathrm{~cm}$ length) were fixed in the center of the bipolar cell. Two graphite feeder electrodes were positioned at the extremities of the cell ( $5 \mathrm{~cm}$ apart). $0.2 \mathrm{M}$ $\mathrm{LiClO} 4$ was used as supporting electrolyte to provide a sufficient amount of ions for charge compensation in the conducting polymer during bipolar actuation in the presence of $5 \mathrm{mM} \mathrm{L-}$ or DDOPA. It is better if the $\mathrm{pH}$ of the sample is around 7 , but an absolute discrimination can also be observed in acidic conditions, considering that the electrooxidation of the two enantiomers of DOPA at $\mathrm{pH} 4$ occurs also at two well separated potentials i.e. $0.6 \mathrm{~V}$ and $0.7 \mathrm{~V}$ vs. SCE. For concentration dependent experiments, 2, 5, 10 and 15 mM L-DOPA solutions were analyzed with a (S)-oligo-(3,3'dibenzothiophene)-polypyrrole hybrid film. For experiments with L- and D-DOPA mixtures, solutions with the enantiomers in $3 \mathrm{mM} / 6 \mathrm{mM}$ and $6 \mathrm{mM} / 3 \mathrm{mM}$ L/D ratios were analyzed with a (S)-oligo-(3,3'dibenzothiophene)-polypyrrole hybrid film. 
The degree of actuation was recorded using a macroscope (LEICA Z16 APO) in video mode.

Movie data treatment was carried out with the help of image J software.

\section{RESULTS AND DISCUSSION}

The overall experimental set-up for absolute wireless chiral discrimination is illustrated in Scheme 2. The hybrid object, composed of enantiopure (R)- or (S)-oligomer BT2T4 (brown) electrodeposited onto Ppy (blue), is used as a bipolar elec-trode, immobilized on an inert support (red). It is mechanically very robust and stable, due to the strong interaction between the conducting oligomer and the polymer. Under the influence of the imposed electric field, the composite bipolar electrode is polarized with respect to the surrounding solution, leading to $\delta+$ and $\delta$ ? extremities. At the $\delta+$ side, electrooxidation of DOPA can occur, for which the enantiopure oligo-BT2T4 provides the chiral selectivity in terms of potential difference. At the $\delta$ ? extremity Ppy is reduced, accompanied by a defor-mation of the polymer following a mechanism described in detail previously.37-40 In order to provide a sufficient amount of electrons for Ppy reduction, the bipolar electrode is de-signed in a way that the $\delta+$ extremity is about 5 times broader than the $\delta$ ? extremity, analog to what has been reported earli-er.41 The reduction of Ppy preferentially occurs on the rough side of the film, facing upwards in the present set-up.38 This is accompanied by an uptake of cations, inducing face selective swelling and thus significant bending.

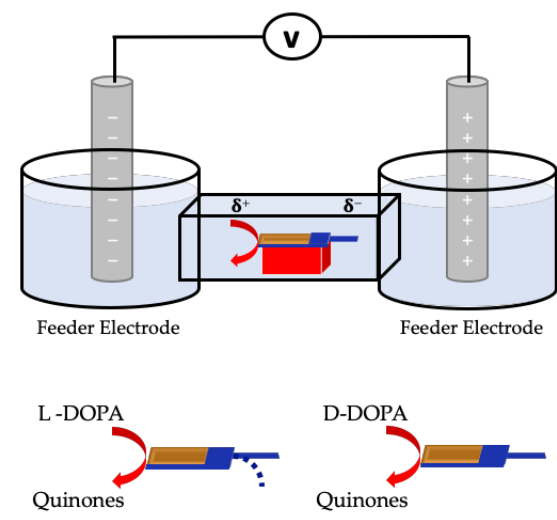

Scheme 2. Schematic illustration of the bipolar cell used for wireless absolute discrimination of Dand L-DOPA. The dark brown part stands for the (S)-BT2T4 oligomer, whereas blue symbolizes the Ppy film. The dotted blue line represents the Ppy bending after enantioselective recognition. The distance between feeder electrodes is $5 \mathrm{~cm}$ and the length of the bipolar electrode is $1.1 \mathrm{~cm}$.

Before performing chiral recognition experiments, it is nec-essary to control and characterize the modification of Ppy with oligo-BT2T4. Surface characterization of the polymer hybrid was performed using scanning electron microscopy (SEM). SEM images were recorded as a top view of three different regions of the bipolar electrode: i) at the junction between Ppy and oligo-BT2T4, ii) at the oligo-BT2T4 layer immobilized on top of Ppy, iii) at the pure Ppy layer. A clear difference in morphology is observed as shown in Figure 1. Oligo-BT2T4 shows a very bright and granular morphology related to the highly conducting surface, whereas the Ppy surface is darker and globular. The bottom side of the hybrid film is uniform, confirming the single side modification of the polypyrrole substrate. Both oligomer enantiomers show exact-ly the same surface morphology when they are deposited on Ppy, which is quite expected as they have the same oligomeri-zation kinetics and contain the same amount of dopant.42 


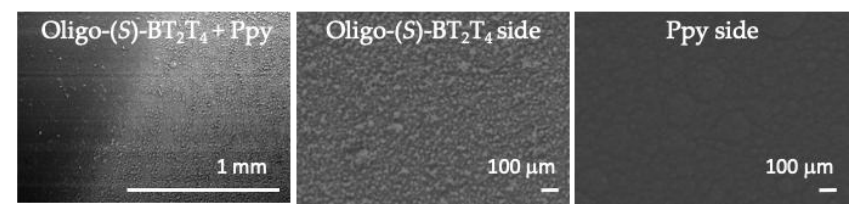

Figure 1. Top view SEM images of the freestanding hybrid film. A) Junction between the oligo-BT2T4 modified part (right) and the unmodified Ppy (left). B) The oligomer layer deposited on the Ppy support. C) the unmodified Ppy film.

Before proceeding to the electromechanical readout exper-iments, chiral recognition tests were performed by differential pulse voltammetry (DPV) using D-DOPA or L-DOPA, re-spectively, in aqueous LiClO4 as supporting electrolyte. The enantioselectivity of the surface of oligo-(S)-BT2T4 with re-spect to D- or L-DOPA is depicted in Figure 2. From the DPV signal it is clear that the electrooxidative transformation of the two enantiomers occurs at significantly different poten-tials i.e. $0.5 \mathrm{~V}$ and $0.7 \mathrm{~V}$ vs. $\mathrm{Ag} \mid \mathrm{AgCl}$ for L-and D-DOPA, respectively. This has already been demonstrated in previous studies 43 and constitutes the crucial ingredient for the absolute chiral discrimination in the follow-up bipolar experiments. It can be clearly seen that at $\sim 0.45 \mathrm{~V}$ vs. $\mathrm{Ag} \mid \mathrm{AgCl}$ only one of the two enantiomers is electrochemically oxidized. Therefore, it should be possible to transform exclusively L-DOPA at a bipolar electrode if the polarization potential difference be-tween its two extremities is tuned at a value that is just enough to allow the oxidation of L-DOPA on one end together with Ppy reduction at the other end. Taking into ac-count that Ppy reduction occurs at about ?0.25 V vs. Ag $\mid \mathrm{AgCl}, 19$ we can conclude that the threshold of such a polarization potential difference should be around $0.65 \mathrm{~V}$.

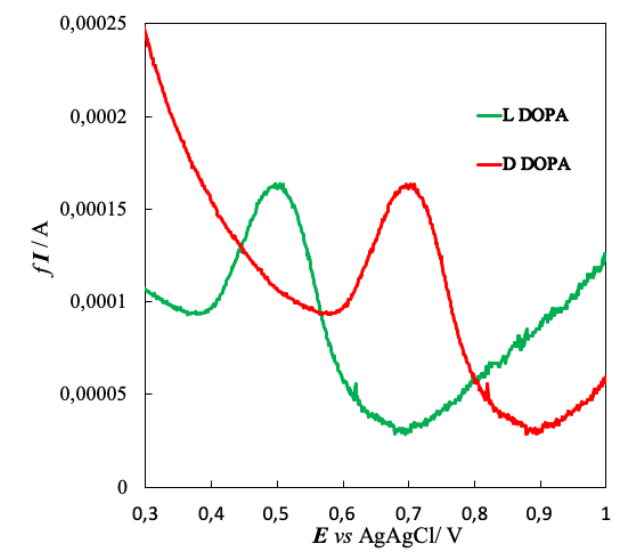

Figure 2. Differential pulse voltammetry in $0.2 \mathrm{M} \mathrm{LiClO4}$ for the enantioselective electrooxidation of Land D-DOPA ( $5 \mathrm{mM}$ ) on the surface at an oligo-(S)-BT2T4 surface, deposited on a free-standing Ppy film.

In order to confirm this value, bipolar electrochemistry was performed by using a hybrid film with oligo-(S)-BT2T4 or oligo-(R)-BT2T4 electrodeposited on Ppy. The potential differ-ence between the feeder electrodes was slowly increased until a first sign of polymer bending was observed. It was found that the minimum electric field required for selective recogni-tion is $0.6 \mathrm{~V} / \mathrm{cm}$. For higher electric fields, both enantiomers can be electrooxidized. It still leads to a certain discrimina-tion, as the rates of bending are different, but prevents from achieving the initial goal, that means absolute recognition of the two enantiomers. When an electric field of $0.6 \mathrm{~V} / \mathrm{cm}$ is applied to the Ppy-based bipolar electrode, modified with one or the other oligo-enantiomer, oligo-(R)-BT2T4/Ppy selectively recognizes D-DOPA, which results in a strong actuation of Ppy at its negatively polarized extremity, 
whereas absolutely no deflection is observed when L-DOPA is in the solution. The opposite effect was observed for oligo-(S)-BT2T4, which is able to recognize L-DOPA, inducing a deflection of Ppy, but remaining electromechanically inactive in the presence of D-DOPA. The difference between the initial (green) and final (red) bending state of the $\delta$ ? extremity of Ppy was used as an output signal during chiral recognition at a fixed time ( $40 \mathrm{~s}$ ) as shown in Figure 3 (see also video V1). The total deflection (= distance between the initial and final position of the pyrrole extremity) in the case of the appropriate enantiomer is about $1 \mathrm{~mm}$, and this kind of deflection is sufficient to classify the device as an optical yes/no reader of chiral information. Ppy might have some issues related to memory effects, but we observed that after switching off the voltage, the Ppy cantile-ver returns back to its original position. Therefore, it is in principle possible to perform several experiments in a row with such a reusable sensor.

Since the discrimination is based on the difference in peak potential values, and not of current intensities, as for most studies presented in literature, there is less risk of interference with other species in the solution, which get oxidized at dif-ferent potentials. However, if a species in solution is much easier to oxidize than the analyte, this will induce an addition-al bending of the polymer and actually lead to an overestima-tion of the real analyte concentration. This is a general prob-lem of most electroanalytical sensors, but doesn't prevent measuring more complex samples. For example, we have car-ried out studies13 employing inherently chiral surfaces for the recognition of L-DOPA in a real matrix (Madopar ${ }^{\circledR}$ tablets used for the treatment of Parkinson's disease). Even in this case LDOPA was successfully discriminated in the presence of other excipients and benserazide, which is also chiral.

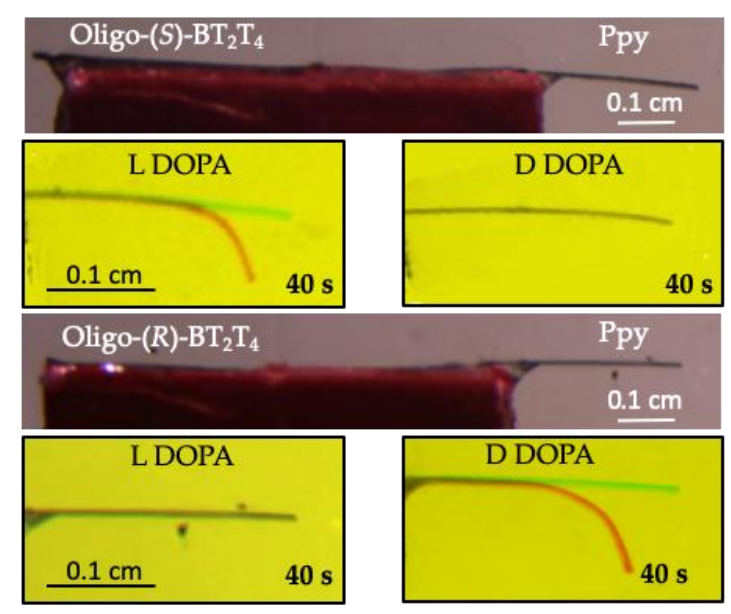

Figure 3. Bipolar enantioselective electrooxidation in $0.2 \mathrm{M} \mathrm{LiClO4}$ of L- and D-DOPA ( $5 \mathrm{mM}$ ) on the surface of oligo-(S)-BT2T4 and oligo-(R)-BT2T4 deposited on Ppy. Initial state (green) and final state (red) of the Ppy extremity during elec-tromechanical deformation. Length of the bipolar electrode is $1.1 \mathrm{~cm}$. The electric field is $0.6 \mathrm{~V} / \mathrm{cm}$ for all experiments. The readout time for all experiments is 40 sec.

Besides this first demonstration of absolute chiral discrimi-nation via an on/off response, we wanted to check in the next step whether the approach can be also adapted to a more quantitative determination of chiral molecules. We therefore measured the degree of deflection as a function of analyte concentration. Four concentrations of L-DOPA $(2,5,10$ and $15 \mathrm{mM})$ were analysed with an oligo-(S)-BT2T4/Ppy bipolar electrode. The degree of deflection was found to be linear in this concentration range (Figure 4). 


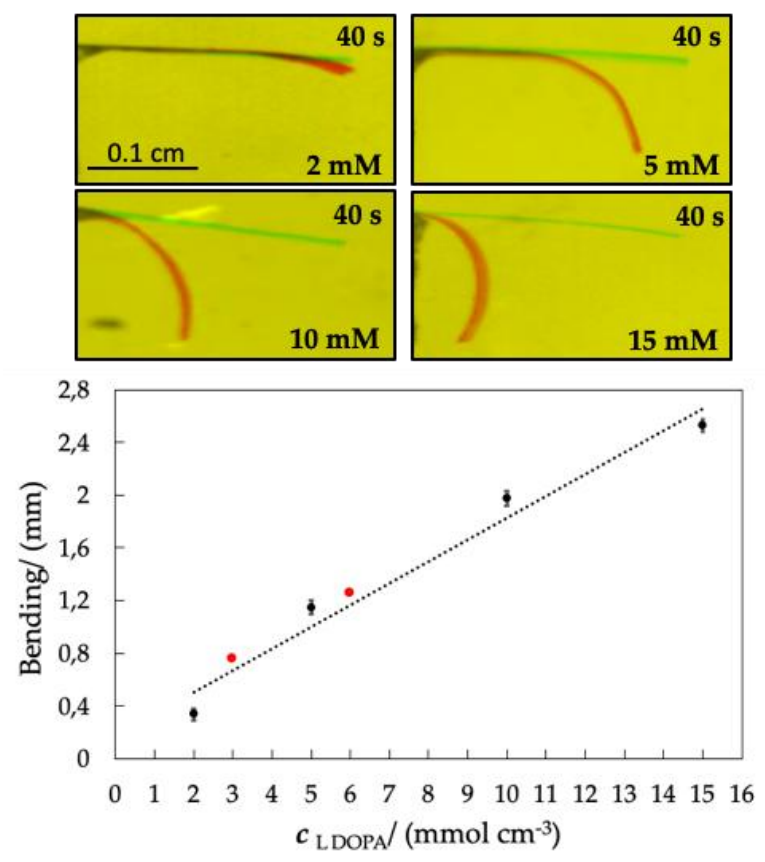

Figure 4. Concentration dependent deflection of Ppy for the enantioselective recognition of L-DOPA on oligo-(S)-BT2T4/Ppy. The readout time for each concentration is $40 \mathrm{sec}$. Length of the bipolar electrode is $1.1 \mathrm{~cm}$. The electric field is $0.6 \mathrm{~V} / \mathrm{cm}$ in all experiments. Error bars correspond to three repetitions. Red dots refer to Ppy deflection for chiral recogni-tion of L-DOPA ( $3 \mathrm{mM}, 6 \mathrm{mM}$, respectively) in the simultaneous presence of D-DOPA $(6 \mathrm{mM}, 3 \mathrm{mM}$, respectively).

After testing the enantioselective deflection as a function of concentration, we have studied this hybrid actuator for the determination of the quantity of only one of the two enantio-mers (independently from the other) when they coexist, in different ratios, in the same solution. We report an example in Figure 5.

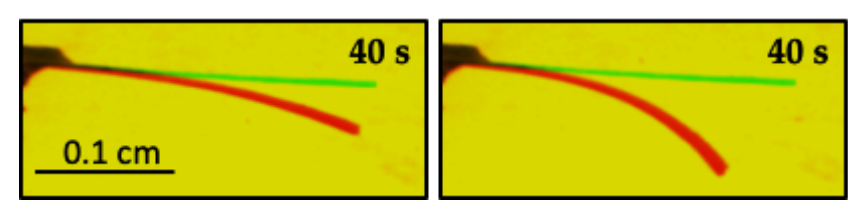

Figure 5. Enantioselective deflection of Ppy for two different solutions containing different ratios of L/D DOPA (3mM/ 6mM left, 6mM/3mM right). Oligo-(S)-BT2T4 was deposited on Ppy. The readout time for each concentration is $40 \mathrm{sec}$. Length of the bipolar electrode is $1.1 \mathrm{~cm}$. The electric field is $0.6 \mathrm{~V} / \mathrm{cm}$ in all the experiments.

The deflection of Ppy varies as a function of the ratio between the two enantiomers of DOPA. When oligo-(S)-BT2T4 is depos-ited on Ppy, the resulting bending is more pronounced in the presence of LDOPA, the favorable enantiomer configuration for selective recognition. The bending response of the hybrid actuator in the presence of the L/D DOPA mixture is coherent with the concentration ratio used during the experiments (red dots in Figure 4: $0.7 \mathrm{~mm}$ vs. $1.3 \mathrm{~mm}$ deflection) and fits the calibration plot. This means that with these experiments it is possible to estimate selectively the concentration of only one of the two enantiomers present in the mixture, the one that is easi-er to oxidize.

Moreover, the enantiomeric excess in mixed solutions of these two enantiomers could be indirectly determined if results from bipolar experiments are combined with another experiment where the total concentration of L- + D-DOPA is correlated to another analytical signal (for example current 
intensities of the chiral analyte recorded in a voltammetry experiment with an achiral electrode substrate like glassy carbon). 44 In this way it could be possible to estimate the total concentration of L- + D-DOPA in solution from a peak current vs. concentration cali-bration plot, while from the bipolar experiments the concentra-tion of one of the two enantiomers (the one that oxidizes first) can be estimated. The enantiomeric excess can then be calcu-lated by combining the two data. Another interesting comple-mentary approach would be to have two cantilevers with oppo-site chirality in the same solution and get a direct read-out of the enantiomeric excess by comparing the deflection ratio of both cantilevers without using any calibration plots. If the con-centrations are very low one still can achieve a visible bending by prolonging the read-out time. So far we always measured the bending after $40 \mathrm{~s}$, because concentrations were in the $\mathrm{mM}$ range. For smaller concentrations, one could read the electro-mechanical signal for example after several minutes and in this way still get significant and reliable bending values. We there-fore anticipate that the method might become very sensitive after an optimization of parameters like read-out time, length and thickness of the cantilever as well as its geometry (ratio between the surface of the part modified with the chiral oligo-mer and the surface of the cantilever). It should be possible to reach sensitivities in the range of a few \%ee, competitive with traditional analytical techniques. However, this would be a project by itself and goes beyond this first proof-of-principle study.

\section{CONCLUSION}

Absolute enantiomeric discrimination has been successfully achieved for the first time with a wireless electromechanical readout concept based on the use of bipolar electrochemistry. A hybrid Ppy film, modified with oligomers bearing intrinsic chiral features, was used as a bipolar electrode. The chronopo-tentiometric surface modification of Ppy with enantiopure oligo-BT2T4 can be optimized by adjusting the experimental conditions (e.g. type of supporting electrolyte, monomer concentration). The morphology of the hybrid film proves to be overall robust and mechanically strong enough to be handled as a free-standing film during the bipolar electrochemistry experiments. Actuation efficiency can be controlled by the applied electric field, allowing addressing selectively only one of the two enantiomers of DOPA as a chiral model analyte. The degree of actuation is found to be directly proportional to the analyte concentration, using both the single enantiomer and mixtures of both enantiomers. We consider the presented bipolar electrochemical approach as very reliable because experiments have been repeated three times for each configu-ration of the BT2T4 films and for each enantiomer of DOPA. The obtained standard deviation of \pm 0.05 confirms the reproducible recognition capabilities of these inherently chiral surfaces. This enables the selective measurement of enantio-mers of chiral probes, both, from a qualitative and quantitative point of

view and, as a consequence, the determination of the enantiomeric excess. Further optimization and generalization will open up interesting perspectives for using this approach as an alternative and straightforward tool to analyze samples containing chiral molecules.

\section{ASSOCIATED CONTENT}

\section{Supporting Information}

The Supporting Information is available free of charge on the ACS Publications website. 


\section{AUTHOR INFORMATION}

Corresponding Author

* Alexander Kuhn a Univ. Bordeaux, CNRS UMR 5255, Bor-deaux INP, ENSCBP, 16 avenue Pey Berland, 33607 Pessac, France, Email: kuhn@enscbp.fr

Present Addresses

Serena Arnaboldi ? Univ. Bordeaux, CNRS UMR 5255, Bordeaux INP, ENSCBP, 16 avenue Pey Berland, 33607 Pessac, France; Univ. degli Studi di Milano, Dip. di Chimica, Via Golgi 19, 20133 Milano; Italy.

Bhavana Guptał ? Univ. Bordeaux, CNRS UMR 5255, Bor-deaux INP, ENSCBP, 16 avenue Pey Berland, 33607 Pessac, France; Engineering Research Center for Nanomaterials, Henan University, Kaifeng 475004, China

Tiziana Benincori ⿶ㅕ][Univ.degli Studi dell'Insubria, Dip. di Scienza e Alta Tecnologia,Via Valleggio 11, 22100 Como, Italy.

Giorgia Bonetti - Univ.degli Studi dell'Insubria, Dip. di Scienza e Alta Tecnologia,Via Valleggio 11, 22100 Como, Italy.

Roberto Cirilli ? Centro Nazionale per il Controllo e la Valutazione dei Farmaci, Istituto Superiore di Sanità, Viale Regina Elena 299, 00161 Roma, Italy.

\section{Author Contributions}

S.A. and B.G. designed and performed experiments; wrote and edited the manuscript. A.K. proposed the research project, provided resources, designed experiments and edited the manu-script.

T.B. designed the inherently chiral monomers, G.B. synthetized the inherently chiral monomers, R.C separated the enantiomers of the inherently chiral monomers by chiral HPLC.

\section{Notes}

The authors declare no competing financial interest.

\section{ACKNOWLEDGMENT}

The work has been funded by the European Research Council (ERC) under the European Union's Horizon 2020 research and innovation program (grant agreement $n^{\circ} 741251$, ERC Ad-vanced grant ELECTRA). This study has also received financial support from the French State in the framework of the "Invest-ments for the future" Program, IdEx Bordeaux, reference ANR-10-IDEX-03-02. S.A. gratefully acknowledges the financial support of Università degli Studi di Milano for a post-doc scholarship. The authors are very grateful for fruitful discus-sions with Patrizia Mussini about this project.

\section{REFERENCES}

1. Arnaboldi, S.; Magni, M.; Mussini, P. R. Enantioselective Selectors for Chiral Electrochemistry and Electroanalysis: Stereogenic Elements and Enantioselection Performance. Curr. Opin. Electrochem. 2018, 8, 60-72. 
2. Wattanakit, C.; Côme, Y. B. S.; Lapeyre, V.; Bopp, P. A.; Heim, M.; Yadnum, S.; Nokbin, S.; Warakulwit, C.; Limtrakul, J.; Kuhn, A. Enantioselective Recognition at Mesoporous Chiral Metal Surfaces. Nat. Commun. 2014, 5, 3325.

3. Stinson, S. C. Chiral Drugs. Chem. Eng. News 1992, 70, 46-79.

4. LaPlante, S. R.; Fader, L. D.; Fandrick, K. R.; Fandrick, D. R.; Hucke, O.; Kemper, R.; Miller, S. P. F.; Edwards, P. J. As-sessing Atropisomer Axial Chirality in Drug Discovery and Develop-ment. J. Med. Chem. 2011, 54, 7005-7022.

5. Navarro-Sánchez, J.; Argente-García, A. I.; Moliner-Martínez, Y.; Roca-Sanjuán, D.; Antypov, D.; Campíns-Falcó, P.; Rosseinsky, M. J.; Martí-Gastaldo, C. Peptide Metal-Organic Frame-works for Enantioselective Separation of Chiral Drugs. J. Am. Chem. Soc. 2017, 139, 4294-4297.

6. Yu, L.-Y.; Liu, Q.; Wu, X.-W.; Jiang, X.-Y.; Yu, J.-G.; Chen, X.-Q. Chiral Electrochemical Recognition of Tryptophan Enan-tiomers at a Multi-Walled Carbon Nanotube-Chitosan Composite Modified Glassy Carbon Electrode. RSC Adv. 2015, 5, 98020-98025.

7. Assavapanumat, S.; Ketkaew, M.; Kuhn, A.; Wattanakit, C. Synthesis, Characterization, and Electrochemical Applications of Chiral Imprinted Mesoporous Ni Surfaces. J. Am. Chem. Soc. 2019, $141,18870-18876$.

8. lacob, B.-C.; Bodoki, E.; Florea, A.; Bodoki, A. E.; Opre-an, R. Simultaneous Enantiospecific Recognition of Several $\beta$-Blocker Enantiomers Using Molecularly Imprinted Polymer-Based Electrochemical Sensor. Anal. Chem. 2015, 87, 2755-2763.

9. Scida, K.; Cunningham, J. C.; Renault, C.; Richards, I.; Crooks, R. M. Simple, Sensitive, and Quantitative Electrochemical Detection Method for Paper Analytical Devices. Anal. Chem. 2014, 86, 6501-6507.

10. Pradela-Filho, L. A.; Noviana, E.; Araújo, D. A. G.; Takeuchi, R. M.; Santos, A. L.; Henry, C. S. Rapid Analysis in Con-tinuous-Flow Electrochemical Paper-Based Analytical Devices. ACS Sens. 2020, 5, 274-281.

11. $\mathrm{Hu}$, J.; Yu, Y.; Brooks, J. C.; Godwin, L. A.; So-masundaram, S.; Torabinejad, F.; Kim, J.; Shannon, C.; Easley, C. J. A Reusable Electrochemical Proximity Assay for Highly Selective, Real-Time Protein Quantitation in Biological Matrices. J. Am. Chem. Soc. 2014, 136, 8467-8474.

12. Sannicolò, F.; Arnaboldi, S.; Benincori, T.; Bonometti, V.; Cirilli, R.; Dunsch, L.; Kutner, W.; Longhi, G.; Mussini, P. R.; Pani-gati, M.; Pierini, M.; Rizzo S. Potential-Driven Chirality Manifesta-tions and Impressive Enantioselectivity by Inherently Chiral Electro-active Organic Films. Angew. Chem. Int. Ed. 2014, 53, 2623-2627.

13. Arnaboldi, S.; Benincori, T.; Penoni, A.; Vaghi, L.; Cirilli, R.; Abbate, S.; Longhi, G.; Mazzeo, G.; Grecchi, S.; Panigati, M.; Mussini, P. R. Highly Enantioselective "Inherently Chiral” Electroac-tive Materials based on a 2,2'-biindole Atropisomeric Scaffold. Chem. Sci. 2019, 10, 2708-2717.

14. Sannicolo', F.; Mussini, P.R.; Benincori, T.; Martinazzo, R.; Arnaboldi, S.; Appoloni, G.; Panigati, M.; Quartapelle Procopio, E.; Marino, V.; Cirilli, R.; Casolo, S.; Kutner, J. W.; Noworyta, K.; Pietrzyk-Le, A.; Iskierko, Z.; Bartold. K. Inherently Chiral Spider-Like Oligothiophenes. Chem. Eur. J., 2016, 22, $10839-10847$.

15. Arnaboldi, S.; Grecchi, S.; Magni, M.; Mussini P. Electro-active Chiral Oligo- and Polymer Layers for Electrochemical Enanti-orecognition. Curr. Opin. Electrochem. 2018, 7, 188-199. 
16. Ainla, A.; Mousavi, M.; Tsaloglou, M.-N.; Redston, J.; Bell, J.; Fernández-Abedul, M. T.; Whitesides, G. Open-Source Potentiostat for Wireless Electrochemical Detection with Smartphones. Anal. Chem. 2018, 90.

17. Bandodkar, A. J.; Jeerapan, I.; Wang, J. Wearable Chemi-cal Sensors: Present Challenges and Future Prospects. ACS Sens. 2016, 1, 464-482.

18. Chow, K.-F.; Chang, B.-Y.; Zaccheo, B. A.; Mavré, F.; Crooks, R. M. A Sensing Platform Based on Electrodissolution of a Ag Bipolar Electrode. J. Am. Chem. Soc. 2010, 132, 9228-9229.

19. Zhang, L.; Gupta, B.; Goudeau, B.; Mano, N.; Kuhn, A. Wireless Electromechanical Readout of Chemical Information. J. Am. Chem. Soc. 2018, 140, 15501-15506.

20. Shi, H.-W.; Zhao, W.; Liu, Z.; Liu, X.-C.; Xu, J.-J.; Chen, H.-Y. Temporal Sensing Platform Based on Bipolar Electrode for the Ultrasensitive Detection of Cancer Cells. Anal. Chem. 2016, 88, 87958801.

21. Mavré, F.; Anand, R. K.; Laws, D. R.; Chow, K.-F.; Chang, B.-Y.; Crooks, J. A.; Crooks, R. M. Bipolar Electrodes: A Useful Tool for Concentration, Separation, and Detection of Analytes in Microelectrochemical Systems. Anal. Chem. 2010, 82, 8766-8774.

22. Zhang, W.; Caldarola, M.; Pradhan, B.; Orrit M. Gold Na-norod Enhanced Fluorescence Enables Single-Molecule Electrochem-istry of Methylene Blue. Angew. Chem. Int. Ed. 2017, 56, 35663569;

23. Guerrette, J. P.; Percival, S. J.; Zhang, B. Fluorescence coupling for direct imaging of electrocatalytic heterogeneity. J. Am. Chem. Soc. 2013, 135, 855-861;

24. Voci, S.; Goudeau, B.; Valenti, G.; Lesch, A.; Jović, M.; Rapino, S.; Paolucci, F.; Arbault, S.; Sojic, N. Surface-Confined Electrochemiluminescence Microscopy of Cell Membranes. J. Am. Chem. Soc. 2018, 140, 14753-14760;

25. Doeven, E. H.; Zammit, E. M.; Barbante, G. J.; Hogan, C. F.; Barnett, N. W.; Francis, P. S. Selective Excitation of Concomitant Electrochemiluminophores: Tuning Emission Color by Electrode Potential. Angew. Chem. Int.I Ed. 2012, 51, 4354-4357;

26. Pinyou, P.; Conzuelo, F.; Sliozberg, K.; Vivekananthan, J.; Contin, A.; Pöller, S.; Plumeré, N.; Schuhmann, W. Coupling of an Enzymatic Biofuel Cell to an Electrochemical Cell for Self-Powered Glucose Sensing with Optical Readout. Bioelectrochemistry. 2015, 106, 22-27.

27 Martinez, J. G.; Otero, T.F.; Jager E.W.H. Effect of the Electrolyte Concentration and Substrate on Conducting Polymer Actuators Langmuir. 2014, 30, 3894 -3904.

28 Garcìa-Còrdova, F.; Valero, L.; Ismail, Y.A.; Otero, T. F. Biomimetic Polypyrrole Based all Three-in-One Triple Layer Sensing Actuators Exchanging Cations. J. Mater.Chem. 2011, 21,17265 17272.

29 Sen, S.G.; Palmore T. R. Stimuli-Responsive Macromolec-ular Composites: Enhanced Stress Modulation in Polypyrrole with Redox-Active Dopants. Macromolecules 2016, 49, 8479-8488.

30 Inagi, S.; Ishiguro, Y.; Atobe, M.; Fuchigami, T. Bipolar Patterning of Conducting Polymers by Electrochemical Doping and Reaction. Angew. Chem. Int. Ed., 122, 10334-10337. 
31 AlTal, F.; Gao, J. Laser-Induced Bipolar Electrochemistry On-Demand Formation of Bipolar Electrodes in a Solid Polymer Light-Emitting Electrochemical Cell. J. Am. Chem. Soc. 2018, 140, 97379742.

$32 \mathrm{Hu}, \mathrm{S}$; Gao, J. Wireless Electroluminescence: Polymer Light-Emitting Electrochemical Cells with Ink-jet Printed 1D and 2D Bipolar Electrode Arrays. J. Phys. Chem. C. 2018, 122, 9054-9061.

33. Jansod, S.; Cuartero, M.; Cherubini, T.; Bakker, E. Color-imetric Readout for Potentiometric Sensors with Closed Bipolar Electrodes. Colorimetric Readout for Potentiometric Sensors with Closed Bipolar Electrodes. Anal. Chem. 2018, 90, 6376-6379.

34. Cunningham, J. C.; DeGregory, P. R.; Crooks, R. M. New Functionalities for Paper-Based Sensors Lead to Simplified User Operation, Lower Limits of Detection, and New Applications. Annu. Rev. Anal. Chem. 2016, 9, 183-202.

35. Chow, K.-F.; Mavré, F.; Crooks, J. A.; Chang, B.-Y.; Crooks, R. M. A Large-Scale, Wireless Electrochemical Bipolar Electrode Microarray. J. Am. Chem. Soc. 2009, 131, 8364-8365.

36. Roark, B.; Tan, J. A.; Ivanina, A.; Chandler, M.; Cas-taneda, J.; Kim, H. S.; Jawahar, S.; Viard, M.; Talic, S.; Wustholz, K. L.; Yingling, Y. G.; Jones, M.; Afonin, K. A. Fluorescence Blink-ing as an Output Signal for Biosensing. ACS Sens. 2016, 1, 1295-1300.

37. Assavapanumat, S.; Gupta, B.; Salinas, G.; Goudeau, B.; Wattanakit, C.; Kuhn, A. Chiral Platinum-Polypyrrole Hybrid Films as Efficient Enantioselective Actuators. Chem. Commun. 2019, 55, 10956-10959.

38. Gupta, B.; Goudeau, B.; Kuhn, A. Wireless Electrochemi-cal Actuation of Conducting Polymers. Angew. Chem. Int. Ed. 2017, 56, 14183-14186.

39. Gupta, B.; Goudeau, B.; Garrigue, P.; Kuhn, A. Bipolar Conducting Polymer Crawlers Based on Triple Symmetry Breaking. Adv. Funct. Mater. 2017, 28, 1705825.

40. Gupta, B.; Afonso, M. C.; Zhang, L.; Ayela, C.; Garrigue, P.; Goudeau, B.; Kuhn, A. Wireless Coupling of Conducting Polymer Actuators with Light Emission. ChemPhysChem 2019, 20, 941-945.

41. Zhan, W.; Alvarez, J.; Crooks, R. M. Electrochemical Sensing in Microfluidic Systems using Electrogenerated Chemilumi-nescence as a Photonic Reporter of Redox Reactions. J. Am. Chem. Soc. 2002, 124, 13265-13270.

42. Paschen, S.; Carrard, M.; Senior, B.; Chao, F.; Costa, M.; Zuppiroli, L. Morphology of a Conducting Polymer and its Relation to the Electronic Properties. Acta Polym. 1996, 47, 511-519.

43. Arnaboldi, S.; Benincori, T.; Cirilli, R.; Grecchi, S.; San-tagostini, L.; Sannicolò, F.; Mussini, P. R. "Inherently Chiral" Thio-phene-Based Electrodes at Work: a Screening of Enantioselection Ability Toward a Series of Pharmaceutically Relevant Phenolic or Catecholic Amino Acids, Amino Esters, and Amine. ABC 2016, 408, 7243-7254.

44. Arnaboldi, S.; Benincori, T.; Cirilli, R.; Kutner, W.; Mag-ni, M.; Mussini, P. R.; Noworyta, K.; Sannicolò F. Inherently Chiral Electrodes: the Tool for Chiral Voltammetry. Chem. Sci. 2015, 6, 17061711. 